\title{
Organização tecnológica do trabalho em um pronto atendimento e a autonomia do trabalhador de enfermagem*
}

\author{
TECHNOLOGICAL ORGANIZATION OF LABOR IN AN EMERGENCY SERVICE \\ AND NURSING WORKER' S AUTONOMY
}

\section{ORGANIZACIÓN TECNOLÓGICA DEL TRABAJO EN UN SERVICIO DE EMERGENCIA Y LAAUTONOMÍA DEL TRABAJADOR DE ENFERMERÍA}

\author{
Giselda Quintana Marques', Maria Alice Dias da Silva Lima²
}

\begin{abstract}
RESUMO
Este estudo teve por objetivo analisar a organização dos processos de trabalho em um serviço de pronto atendimento e a autonomia do trabalhador de enfermagem na prestação de cuidados ao usuário. Tratase de um estudo de caso, com abordagem qualitativa. Os dados foram coletados por meio de observação livre por amostragem de tempo. O foco de observação foi o atendimento prestado aos usuários no serviço. Constatou-se que o processo de trabalho está organizado com a finalidade de tratar a queixa principal, tendo como ação nuclear a consulta médica. A organização tecnológica do trabalho coloca o médico como detentor de poder, seguido da enfermeira, nos seus espaços de poder e autonomia, que é pouco exercida pelos demais agentes. $O$ trabalho da enfermagem é fundamental, perpassando todos os espaços de atendimento e interligando as ações, mas se caracteriza como um trabalho auxiliar das atividades do pronto atendimento.
\end{abstract}

\section{DESCRITORES}

Serviços de Saúde.

Qualidade da Assistência à Saúde.

Serviços Médicos de Emergência.

Enfermagem.

Autonomia profissional

\section{ABSTRACT}

This research was aimed at examining the organization of labor processes in an emergency service as well as the autonomy nursing workers have when providing care. It is a qualitatively-oriented case study. Data were collected by free observation, with focus on the service provided to users. It was observed that the labor process is organized around the treatment of the main complaint, having the doctor's visit as its core action. Technological organization places doctors as the main power holders, followed by nurses in their spaces of power and autonomy - an autonomy that is seldom exercised by the remaining agents. Nurses' work is crucial, traversing all spaces of service and interlinking actions; however, it is seen as supplementary to primary care activities.

\section{KEY WORDS}

Health Services.

Quality of Health Care.

Emergency Medical Services.

Nursing.

Professional autonomy

\begin{abstract}
RESUMEN
El estudio tiene por objetivo analizar la organización de los procesos de trabajo en un servicio de emergencia y la autonomía del trabajador de enfermería en la prestación de cuidados al usuario. Se trata de un estudio de caso con abordaje cualitativo. Los datos fueron recogidos por medio de observación libre por muestreo de tiempo. El foco de observación fue la atención prestada a los usuarios en el servicio de emergencia. Se constató que el proceso de trabajo está organizado con la finalidad de tratar la queja principal, teniendo como acción nuclear la consulta médica. La organización tecnológica del trabajo coloca al médico como detentor del poder, seguido por la enfermera, en sus espacios de poder y autonomía, autonomía que es poco ejercida por los demás agentes. El trabajo de enfermería es fundamental, siguiendo todos los espacios de la atención e interconectando las acciones, no obstante se caracteriza como un trabajo auxiliar de las actividades de emergencia.
\end{abstract}

\section{DESCRIPTORES}

Servicios de Salud.

Calidad de la Atención de Salud. Servicios Médicos de Urgencia.

Enfermería.

Autonomía profesional

\footnotetext{
* Extraído da dissertação"Demandas do pronto atendimento e os processos de trabalho em serviços de porta aberta", Escola de Enfermagem, Universidade Federal do Rio Grande do Sul, 2004. ${ }^{1}$ Enfermeira da Secretaria Municipal da Saúde de Porto Alegre. Doutoranda em Enfermagem da Escola de Enfermagem, Universidade Federal do Rio Grande do Sul. Membro do Grupo de Estudos em Saúde Coletiva (GESC), Universidade Federal do Rio Grande do Sul (UFRS). Porto Alegre, RS, Brasil. gqmarques@terra.com.br ${ }^{2}$ Doutora em Enfermagem. Professora Associada da Escola de Enfermagem, Universidade Federal do Rio Grande do Sul. Membro do Grupo de Estudos em Saúde Coletiva (GESC), Universidade Federal do Rio Grande do Sul (UFRS). Porto Alegre, RS, Brasil. malice@enf.ufrgs.br
} 


\section{INTRODUCÃ̃}

Na década de 1990, com as mudanças na legislação do Sistema Único de Saúde, trabalhadores e instituições são instigados a reorganizar seus processos de trabalho, em busca da integralidade das ações. A partir daí, com essas mudanças, a saúde passa a ser um direito do cidadão e um dever do Estado, com acesso universal e igualitário, sendo que as relações de trabalho devem ser mais horizontais.

Apesar das mudanças instituídas pelos gestores, nos últimos anos, estudos têm mostrado que o resultado obtido ainda está aquém do esperado, tanto pelos gestores, trabalhadores, quanto usuários ${ }^{(1-5)}$. Uma das justificativas para tal é que os processos de trabalho ainda são fortemente influenciados por saberes, equipamentos, normas e estruturas organizacionais, dando-se ênfase aos aspectos biológicos para interpretação dos fenômenos vitais, aos procedimentos, ao saber médico e às especialidades (modelo biomédico). Esse desenho tecno-assistencial está organizado na divisão de tarefas entre os diferentes agentes, tendo o médico como o centro da assistência. A inserção do usuário no Sistema é feita pelo diagnóstico médico da doença (queixa-conduta). Os demais agentes entram em ação, a partir de um indicativo desse profissional, com ações complementares.

A organização dos processos de trabaIho se constitui pelos seus elementos: o objeto de trabalho, os meios de produção e o trabalho humano, assim como as relações técnicas, sociais e de produção. Historicamente, por meio do trabalho, os seres humanos produzem e reproduzem a sua existência, tanto pelos processos objetivos quanto subjetivos ${ }^{(3,6)}$.

O produto final do trabalho na saúde, o cuidado, é consumido, pelo usuário, no mesmo momento em que é produzido; sendo assim, é indissociável do processo que o produziu, é a própria realização da atividade ${ }^{(7)}$.

O processo de trabalho é determinado pela sua finalidade, pela idealização do resultado que foi projetado, pelo trabalhador, muito antes da sua realização. $O$ produto obtido contém a intenção desse trabalhador que efetua a ação, cujo objeto, o usuário, também interage no processo, colocando nesse espaço a sua intencionalidade, conhecimento e representações. Nessa troca de subjetividades está expressa a concepção de saúde e doença de quem produz e de quem recebe os cuidados, assim como o modo de produção dos mesmos ${ }^{(7)}$.

A mudança de paradigma, do centrado na doença para o das necessidades em saúde, não dependerá somente do desejo de cada trabalhador/gestor/usuário, mas da lente que será usada para observar e transformar o fenômeno. Como em um caleidoscópio em que as partes que o compõem estão sempre presentes, o que diferencia um desenho do outro é o arranjo das peças; na Saúde, os elementos do processo de trabalho também estão sempre presentes, o produto da ação do trabalhador dependerá do rearranjo dos diferentes elementos, da ênfase que será dada às necessidades do usuário e ao ordenamento das tecnologias.

Em todo o processo de trabalho está presente um quantum de dominação e controle, o que garante margens de negociação entre os agentes. A organização cientifica dos processos de trabalho, com divisão rígida de tarefas e desvalorização dos conhecimentos e das vivências dos trabalhadores tem levado à alienação e à conseqüente desresponsabilização pelo atendimento.

Tanto no cenário do hospital quanto no de Saúde Pública a Enfermagem e as demais profissões têm trabaIhado com autonomia relativa, sendo modeladas e orientadas pelo trabalho médico. A Enfermagem, por desenvolver um trabalho muito interligado ao do médico, dando ênfase às tarefas e aos procedimentos, tende a obedecer às demandas desse profissional em detrimento das apresentadas pelo usuário. Esse processo, manual e repetitivo, produz um trabalho esvaziado de conteúdo científico que ao longo do tempo aliena o trabalhador.

No desempenho da ação, o profissional poderá tanto usar o que já está construído, estabelecido, como pode exercer a sua autonomia, construindo novas formas de cuidado em saúde. Sempre existirá um espaço de exercício de autonomia do trabalhador, pois o trabalho vivo em ato pressupõe a possibilidade de criatividade, podendo ser explorada para inventar novos processos de trabalho, inclusive para aquelas dimensões ainda não pensadas. Mesmo nos processos mais arraigados, sempre há a possibilidade de se formarem fissuras para que seja questionada a lógica estruturada de produção de cuidados, podendo, assim, o trabalhador, estabelecer linhas de fuga que ampliem a sua atuação e autonomia em prol da satisfação das necessidades do usuário(7).

A justificativa para este estudo parte do pressuposto de que a enfermagem tem utilizado uma pequena margem de liberdade no seu trabalho, sendo fortemente influenciada pelos saberes biológicos, regras/normas instrumentais da profissão e tendo o médico como a figura chave no atendimento em saúde.

Com base no acima descrito, tem-se como objetivo analisar a organização dos processos de trabalho em um pronto atendimento e a autonomia do trabalhador de enfermagem na prestação de cuidados ao usuário. 


\section{MÉTODO}

É uma pesquisa com abordagem qualitativa, que apreende a realidade e compreende os fenômenos e processos sociais que se manifestam no cotidiano do trabalho e que têm reflexo direto e indireto na vida das pessoas que buscam e que recebem atendimento em saúde. Favorece a observação de vários elementos, de forma simultânea, buscando entender e descrever o contexto onde o fenômeno ocorre ${ }^{(8)}$. O desenho metodológico da pesquisa é o estudo de caso, que permite um aprofundamento da unidade estudada, vista na sua singularidade ${ }^{(9)}$.

A coleta de dados foi realizada por meio da observação livre, por amostragem de tempo ${ }^{(10)}$, em um Serviço de Pronto Atendimento de um Centro de Saúde, gerenciado pelas diretrizes estratégicas da Secretaria Municipal da Saúde (SMS) de Porto Alegre. O foco de observação foi o atendimento prestado aos usuários, destacando a organização do processo de trabalho da enfermagem, no período de agosto a dezembro de 2003.

A observação foi realizada na sala de espera, na recepção, no espaço da pré-consulta, no corredor de espera, em frente aos consultórios, na sala de observação e de procedimentos. A maior parte da observação se concentrou no espaço da pré-consulta e na sala de observação. Esses dois locais, por serem abertos e amplos, proporcionam a visão de outros espaços de atendimento, sendo possível observar o seu funcionamento. Na sala de observação são realizados quase todos os procedimentos, tanto da enfermagem, quanto dos médicos. Os períodos de observação ocorreram em horários escalonados, de forma intencional, nos dias da semana e nos três turnos de trabalho.

O projeto foi aprovado pelo Comitê de Ética em Pesquisa da SMS/POA (CEP no 021/2003). Aos trabalhadores de saúde foi entregue o Termo de Consentimento Livre e Esclarecido, o qual respeita os aspectos éticos apontados pela resolução 196/96 do Conselho Nacional de Saú$\mathrm{de}^{(11)}$. Para garantia do anonimato e da confidencialidade dos dados, ao se fazer referência aos trabalhadores de enfermagem utiliza-se o sexo feminino, por serem em maior número, e para os profissionais médicos, o sexo masculino.

A análise dos dados foi realizada, seguindo diretrizes do método qualitativo: ordenação, classificação em estruturas de relevância, síntese e interpretação ${ }^{(8)}$. A etapa de classificação foi realizada com os dados obtidos nas observações registradas no diário de campo, os quais foram transcritos e transportados para o software Ethnograph versão 5.0 (Qualis Research Associates), que foi utilizado para a operacionalização da análise, agilizando a organização do material em estruturas de relevância. A interpretação possibilitou uma visão mais ampla do trabalho realizado no serviço, assim como destacou a dimensão do que é desenvolvido pela enfermagem, permitindo vislumbrar algumas estratégias de ação que levam em conta as margens de liberdade e o potencial do trabalhador daquele serviço.

\section{RESULTADOS E DISCUSSÃO}

\section{A organização tecnológica do trabalho no Serviço de Pronto Atendimento}

O Serviço de Pronto Atendimento (SPA) está vinculado administrativamente à Unidade Básica de Saúde (UBS), de um Centro de Saúde, que foi construído na década de 1970, pela esfera federal, tendo sido concebido como um grande Ambulatório de Especialidades Médicas. Na municipalização, em 1996, houve a reorganização do acesso pelo pré-agendamento de consultas, hierarquização dos serviços, passando a Centro de Saúde de atenção básica (UBS e Pronto Atendimento) e de média complexidade (Ambulatório de Especialidades).

Apesar das mudanças administrativas e políticas estabelecidas pelo Gestor Público, a organização tecnológica do SPA ainda é fortemente influenciada pelo modelo biomédico. O acesso da população ao serviço é feito, primeiramente, pelo preenchimento de um boletim de atendimento, para posterior avaliação pelas técnicas/auxiliares de enfermagem, na pré-consulta; essa avaliação determina o tipo de atendimento a ser utilizado. Os critérios de acesso e de prioridade são preestabelecidos pela Instituição/Serviço e por critério do profissional, no seu turno de trabalho.

No modelo biomédico, o enfoque dado pelo trabalhador é no procedimento; o corpo anatomo-biológico é identificado pela queixa que o usuário traz, na tentativa de transformá-la em um possível diagnóstico; sendo assim, a queixa deixa de fazer parte da pessoa e se transforma em um sinal ou sintoma de doença, que, então, será o objeto de trabalho do médico e da enfermagem ${ }^{(12)}$.

Constatou-se que é freqüente a população usuária utilizar a porta da urgência, não só para os casos agudos, mas também, de forma eletiva, para complementar os atendimentos das Unidades Básicas de Saúde e das Unidades Especializadas. Também é freqüente, no discurso dos trabalhadores, que a utilização indevida, nos casos eletivos, descaracteriza a missão de atendimento de urgência, o que coloca o usuário em uma situação de ter que justificar a sua necessidade para ser atendido.

Esse sentimento de desperdício da vocação do serviço, que seria a de tratar somente a urgência e de subtilização do alto e específico preparo técnico dos trabalhadores também faz parte do discurso de trabalhadores de outros serviços de emergência quando dizem que as demandas não urgentes trazem sobrecarga ao trabalho já estressante $\mathrm{e}^{(3,13-15)}$.

Uma porta de entrada organizada para acolher o usuário faz dessa distorção uma agenda de discussão, levan- 
do em conta que existe uma demanda reprimida que bate à porta dos serviços de pronto atendimento e que precisa de respostas às suas necessidades. Ao invés disso, penaliza-se a vítima pelo uso indevido do recurso de saúde, fazendo-a peregrinar.

O preenchimento do boletim de atendimento é manual, feito na recepção do SPA, onde se encontram as folhas em branco, colocadas em uma prancheta, à disposição do usuário. Pela manhã a funcionária administrativa orienta o seu preenchimento. Nos turnos da tarde e noite, os usuários são orientados pela enfermagem. Não existe um funcionário escalado para esta função. Muitas vezes o próprio usuário preenche, sozinho, o boletim, o que por vezes, Ihe ocasiona esperas desnecessárias, nas situações em que a sua demanda não corresponde ao que é ofertado pelo serviço. Os boletins preenchidos são separados em três escaninhos: atendimento clínico, pediátrico e de enfermagem. Por vezes é o vigilante quem faz a primeira abordagem do cliente, na entrada do serviço, fato esse também identificado por outros estudos da Saúde $\mathrm{e}^{(3,13,16)}$.

A recepção pouco qualificada denota que a centralidade do atendimento não é a pessoa e suas necessidades e, sim, a diminuição da demanda. Entretanto, a recepção de um serviço de Saúde é um ponto estratégico. Por ela passam todos os usuários que necessitam de cuidados urgentes ou não; por isso deve ser pensada de forma que consiga dar respostas qualificadas ao usuário ${ }^{(17)}$. É nesse local que ele espera ter sua demanda acolhida, o que não significa solucionar todos os problemas que chegam ao serviço, mas a atenção dispensada na relação de atendimento, que envolve a escuta, a responsabilização e o respeito à pessoa que porta a necessidade.

No SPA são realizados atendimentos referentes à enfermagem, pediatria e clínica e os de sala de observação e reanimação. Os procedimentos de enfermagem para aplicação de injetáveis, nebulizações e glicemia capilar são realizados mediante a apresentação da prescrição médica. Os demais fazem parte da dinâmica dos atendimentos que são oferecidos somente em dias úteis, sendo que os clínicos e de enfermagem são prestados das 7 às 20 horas e 0 atendimento médico-pediátrico, no período das 7 às 18 horas.

A equipe é composta de vigilante, motorista, funcionária administrativa, que atende no guichê de recepção, somente no turno da manhã, de enfermeiras, técnicos/auxiliares de enfermagem, médicos clínicos, médicos pediatras, auxiliares de limpeza e da coordenadora do serviço, que faz parte da escala de plantonistas médicos.

O fluxo de atendimento do usuário inicia após o preenchimento do boletim de atendimento. Dependente da demanda e do critério de gravidade, ele aguarda, ou não, no saguão, o chamado da enfermagem para a segunda triagem, que ocorre no espaço da pré-consulta. Ela serve para ouvir a história do paciente, definir a queixa princi- pal e estabelecer as prioridades de atendimento. Fazem parte dessa avaliação a verificação da pressão arterial e da temperatura axilar, a investigação do motivo do atendimento, da queixa principal e os sinais de gravidade que podem definir a tomada de decisão. Se as necessidades são urgentes, os pacientes são encaminhados diretamente para a sala de observação ou consultório; se não, voltam para a sala de espera e aguardam o chamado para consulta nos blocos de consultas eletivas.

As ações de recepção, triagem e consulta médica fazem parte da centralidade desse serviço. O trabalho da enfermagem é operado com a finalidade de conter a demanda espontânea que é sempre maior do que a capacidade do serviço em atendê-la. O trabalho é realizado muito rapidamente, por vezes, de forma confusa, com diálogos dirigidos para a queixa, podendo trazer um nível de insatisfação grande para usuários e trabalhadores. O trabalho das diferentes categorias profissionais está articulado para garantir o pronto atendimento. Os médicos fazem a consulta, buscando a resolução dos sintomas referidos pelos pacientes e a enfermagem executa atividades a fim de organizar os usuários, conforme a consulta médica.

Essa lógica de produção de cuidados, também faz com que os trabalhadores tratem o indivíduo apenas pela doença, de tal forma que todos são atendidos da mesma maneira, sem que os fatores sociais, econômicos, o sofrimento físico e psíquico, singulares a cada pessoa, sejam valorizados e façam parte do atendimento.

\section{Processo de trabalho da enfermagem e a autonomia do trabalhador}

A autonomia profissional da enfermagem tem sido, ao longo do tempo e da evolução da própria profissão, um tema relevante para a sua compreensão. A autonomia técnica dos profissionais no processo de trabalho é definida como a liberdade de julgamento e tomada de decisão frente às necessidades de saúde dos usuários, que se apresentam no cotidiano(18). A autonomia profissional da enfermagem tem caráter processual e é representada como algo que está sendo construído e que tem relação com as conquistas tecnológicas da profissão(19).

No SPA a escala de enfermagem é organizada com divisão de tarefas entre as técnicas/auxiliares de enfermagem, sendo que o trabalho é dividido entre as que ficam na pré-consulta e as da Sala de Observação (SO). Existe também a figura do volante para aplicação de injetáveis, nebulizações e para os atendimentos de urgência. Na divisão do trabalho, é a enfermagem que recebe os pacientes vindos dos consultórios médicos para administração de medicamentos.

Como não existe um médico escalado para prestar atendimento exclusivo, na sala de observação, mesmo quando há dois médicos na escala de plantão, muitas vezes é a enfermagem que se desloca até os consultórios ou sala de 
descanso para solicitar ao médico a reavaliação de pacientes da SO. Na maioria das vezes, essa atitude está ligada à liberação de vagas e agilização do atendimento.

São, também, as técnicas/auxiliares de enfermagem que se responsabilizam por controlar e chamar os blocos de consultas clínicas e pediátricas. Elas organizam as planilhas, controlam os nomes de quem já foi chamado, ou não, os escaninhos do guichê de recepção, acompanham a agilidade e os fluxos de atendimento, registram as desistências e os nomes de quem está ou esteve em atendimento na SO e orientam os pacientes na pós-consulta.

$\mathrm{Na}$ falta de uma ou mais técnicas/auxiliares, as atividades são reorganizadas, sendo que desaparece a função de volante e é a técnica/auxiliar de enfermagem da SO ou da pré-consulta, dependendo das demandas desses setores, quem assume as nebulizações. Para preenchi-mento da escala de trabalho das 7 às 20 horas, existe escalonamento de horários na enfermagem, preenchendo as necessidades do serviço e do trabalhador.

Na divisão de tarefas, nos turnos, a enfermagem também é responsável pela revisão e reposição dos consultórios, pela limpeza e guarda dos materiais, arrumação dos leitos, reposição de insumos e materiais, pela abertura do serviço, às 7 horas, e pelo fechamento, às 20 horas. 0 médico não participa dessas atividades, iniciando o trabaIho na presença da enfermagem e saindo, quando completa o seu turno de trabalho.

As enfermeiras atuam em todas as frentes de trabalho. Elas coordenam as atividades da enfermagem, articulam, supervisionam e controlam as dinâmicas de trabalho no serviço, assim como selecionam pacientes de maior risco, dentro das prioridades estabelecidas. Também fazem a interligação do trabalho médico com os demais trabalhadores, setores e serviços.

Pode-se afirmar que o seu papel na equipe é indiscutível e imprescindível. O trabalho das enfermeiras é muito interrompido, transitam constantemente pela unidade, sendo solicitadas por todos os profissionais da equipe, para informar familiares sobre o estado de pacientes, organizar a seqüência de atendimentos, autorizar determinado procedimento ou tomada de decisão. São elas que respondem pela manutenção, almoxarifado, farmácia, entre outros, assim como atendem profissionais de outras Instituições, o que já foi bem documentado por autores que estudaram o trabalho da enfermeira ${ }^{(5,12,20-21)}$.

Destaca-se que faz parte do trabalho da enfermeira a resolução de problemas do cotidiano, assim como a garantia da infraestrutura e da manutenção do cuidado, ocupando assim, um espaço vazio na divisão técnica do trabalho em saúde. Esse trabalho sofisticado, difícil e, por vezes, desgastante, tem sido exercido por ela de forma mais eficaz do que pelos demais trabalhadores.

Há diferença na forma como as enfermeiras organizam o trabalho; todas fazem a função de supervisão e gerenciamento, com relação à assistência, cada uma estabelecendo diferentes prioridades no atendimento; uma ocupa-se com o apoio e a assistência direta de casos agudos e urgentes, atuando na recepção e na triagem, quando chamada para avaliação; outra realiza atendimentos pontuais na assistência, circulando em vários locais de atendimento, a terceira circula por todos os âmbitos, sendo a que estabelece relações mais acolhedoras e de inclusão do usuário no centro do cuidado.

Apesar de todo o trabalho que cabe à enfermagem nesse serviço, ela o desenvolve com relativa autonomia. A enfermeira atua em um âmbito maior, transitando melhor entre as diferentes disciplinas/saberes, mas as técnicas/ auxiliares de enfermagem estão presas às normas (formais/informais) e ao saber da enfermagem.

Sendo assim, é a enfermeira que utiliza melhor o espaço de negociação. Nas vezes em que ela leva a discussão para o médico, as demandas são atendidas, o que nem sempre acontece com as técnicas/auxiliares. Pode-se supor que as enfermeiras utilizam com melhor qualidade o seu saber/fazer para defender idéias ou propostas, o que lhes dá maior autonomia, enquanto as auxiliares usam abordagens pouco argumentativas e centradas no fazer da enfermagem.

Nos procedimentos de enfermagem é que se identifica uma maior margem de liberdade no trabalho das técnicas/auxiliares de enfermagem, quando conseguem estabelecer relações mais livres, utilizando um saber tecnológico, construído social e historicamente. No exercício de uma ação concreta, o procedimento de enfermagem, é que parece haver a identificação do cuidado. Esse fato tem alicerces na rigidez da organização científica do processo de trabalho, que valoriza o procedimento e desqualifica o conhecimento, as experiências e a subjetividade dos agentes, principalmente os de nível médio.

O saber é ferramenta importante para o estabelecimento da autonomia profissional, visto que um novo saber abre possibilidades de relações de poder, o qual não será possível ser exercido sem a sua sustentação. Outro elemento central que demonstra o caráter processual da autonomia em enfermagem é a responsabilidade, indicando um comportamento pessoal e profissional necessário para a sua obtenção ${ }^{(19)}$.

O trabalho em saúde sustenta-se pela atividade intensiva, que articula um relativo nível de domínio do seu processo que é desigualmente distribuído entre seus agen- 
tes $^{(3,11)}$, quanto mais dividido e subordinado for o trabaIho, menor será o controle de seu processo. Então, quanto maior for a integralidade do atendimento mais ampla será a margem de liberdade de seus agentes. Isso explica a maior autonomia dos médicos e enfermeiras dentro de seus espaços de atuação, diferentemente das técnicas/ auxiliares de enfermagem.

A fragmentação e segmentação das ações separam o trabalhador do seu produto, acarretando atos esvaziados de sentido e com conseqüente desresponsabilização do trabalhador pelo cuidado prestado. O resultado da alienação é o descaso e a desumanização no atendimento, com conseqüente diminuição da resolutividade.

À medida que os trabalhadores assumem atitudes comprometidas e se inserem criticamente na realidade estão transformando o seu cotidiano, pois, como já foi dito anteriormente, é pelo trabalho que os sujeitos sociais produzem e reproduzem a sua existência, tanto no plano material quanto subjetivo, ambos condicionando-se mutuamente ${ }^{(3,6)}$.

Nesse sentido, a enfermagem, por ter um papel social relevante e um saber tecnológico especifico, pode construir processos de trabalho com maior autonomia e menor subordinação de seus agentes, pois ao mesmo tempo em que tem se subordinado ao trabalho médico, também tem reproduzido essa subordinação dentro de sua equipe. $O$ trabalho da enfermagem não é somente cumprir rotinas e executar tarefas, faz parte de um trabalho coletivo em que os profissionais se inter-relacionam com seus pares e com o usuário no atendimento em saúde.

Os trabalhadores de enfermagem do SPA buscam soluções, tanto padronizadas quanto criativas que atendam às demandas de quem procura por atendimento, mas elas ainda são muito tênues se comparadas ao potencial de governabilidade e de transformação que é possível estabelecer, a partir do cotidiano de seu trabalho, ou seja, de quanto podem ser capazes de intervir na qualidade das ações desenvolvidas e na resolutividade do serviço, atuando como co-gestores de seus processos de trabalho.

No trabalho coletivo sempre há espaço para o diálogo, para a reflexão, tomada de decisão conjunta, na busca de um objetivo único que é a qualidade do cuidado prestado. A comunicação e a interação são potentes ferramentas desse trabalho, por meio do qual o trabalhador introduzirá mudanças na relação de poder existente, tanto entre categorias profissionais, quanto entre profissional-usuário.

\section{CONSIDERAÇÕES FINAIS}

No SPA, a enfermagem atua de forma a garantir o pronto atendimento e organizar a demanda ao médico. Assim, esse profissional fica bastante protegido das mazelas da recepção e da triagem. As demandas que chegam para ele já estão organizadas pela enfermagem. Nesse processo, são as enfermeiras e as técnicas/auxiliares de enfermagem que, inúmeras vezes, exercem a função de barreira, contendo a demanda, administrando conflitos, servindo de guardiãs das normas e da política institucional.

O trabalho está organizado com base na consulta médica. Sua finalidade é recuperar o corpo doente e tratar a doença, pinçada pela queixa principal. O médico, pelo exercício da autonomia e da autoridade que lhe são atribuídas, estipula, de forma marcante, os limites de autonomia dos demais trabalhadores.

Apesar disso, há movimentos para maior autonomia dos profissionais de enfermagem, mas esse movimento ainda é muito tênue e relacionado ao trabalho das enfermeiras. A busca de maior autonomia no trabalho da enfermagem ainda precisa ser identificada por todos como uma estratégia possível, que amplia o acesso dos usuários e diminui as demandas por atendimentos médicos. Assim, a enfermagem precisa assumir seu núcleo de competência, o cuidado, que se volta para a pessoa e não para a doença, utilizando todo o seu saber e sua sensibilidade no desempenho de sua autonomia.

As demandas eletivas simples podem ser acolhidas, de forma responsável, por qualquer membro da equipe, principalmente pela enfermagem, que desempenha um importante papel, que pode ser ampliado por meio de uma escuta mais qualificada, e assim, seu trabalho não se reduz à mera execução da prescrição médica e à realização de normas e rotinas.

Foi evidenciado que a enfermeira tem um papel fundamental e indispensável nesse serviço, articulando os diversos trabalhos, papel esse que é reconhecido e valorizado pelos demais trabalhadores, no momento em que ela é muito solicitada durante o seu turno. Embora pareça-nos uma anomalia, o trabalho da enfermeira tem sido também, o de resolver problemas, das mais variadas ordens. Esse trabalho difícil, sofisticado e, por vezes, desgastante, tem feito parte do dia-a-dia dessa profissional que responde a esta demanda de forma mais eficaz que os demais trabalhadores. Sendo assim, as enfermeiras têm assumido, na divisão técnica do trabalho em saúde, o gerenciamento do cuidado. Cabe salientar que para a coordenação das atividades necessárias para a produção de cuidados é preciso conhecimento, responsabilidade e capacidade de decisão.

A supremacia do médico e o poder que detém são inegáveis, mas o domínio profissional pode ser rompido. Sua autonomia também é relativa e é definida pelos limites de competência e dos saberes impostos pelos outros agentes e pelas relações sociais mais amplas.

A enfermagem, nos serviços de saúde, mantém uma contínua convivência com os usuários, de modo que isso Ihe possibilita grandes oportunidades de interação. Entretanto, sua capacidade para ouvir ainda é limitada, pre- 
cisando desenvolver essa habilidade, bem como a de aproximar-se do cliente, tendo a sensibilidade de identificar, no outro, os aspectos verbais e as expressões corporais, aspectos que a instiguem na procura do autoconhecimento.

\section{REFERÊNCIAS}

1. Cecílio LCO. Modelos tecno-assistenciais em saúde: da pirâmide ao círculo, uma possibilidade a ser explorada. Cad Saúde Pública. 1997;3(13):469-78.

2. Schimith MD, Lima MADS. Acolhimento e vínculo em uma equipe do Programa de Saúde da Família. Cad Saúde Pública. 2004;20(6):1487-94.

3. Deslandes SF. Frágeis deuses: profissionais da emergência entre os danos da violência e a recriação da vida. Rio de Janeiro: FIOCRUZ; 2002.

4. Marques GQ. Demandas do pronto atendimento e os processos de trabalho em serviços de porta aberta [dissertação]. Porto Alegre: Escola de Enfermagem, Universidade Federal do Rio Grande do Sul; 2004

5. Rossi FR, Lima MADS. Fundamentos para processos gerenciais na prática do cuidado. Rev Esc Enferm USP. 2005;39(4):460-8.

6. Ribeiro EM, Pires D, Blank VL. A teorização sobre processo de trabalho em saúde como instrumental para análise do trabalho no Programa Saúde da Família. Cad Saúde Pública. 2004; 20(2):438-46.

7. Merhy EE. Saúde: a cartografia do trabalho vivo. São Paulo: Hucitec; 2002.

8. Minayo MCS. O desafio do conhecimento: pesquisa qualitativa em saúde. 2a ed. São Paulo: Hucitec; 1993.

9. Victora CG, Knauth DR, Hassen MN. Pesquisa qualitativa em saúde: uma introdução ao tema. Porto Alegre: Tomo Editorial; 2000.

10. Triviños ANS. Introdução à pesquisa em ciências sociais: a pesquisa qualitativa em educação. São Paulo: Atlas; 1987.

11. Conselho Nacional de Saúde. Resolução n. 196, de 10 de outubro de 1996. Dispõe sobre diretrizes e normas regulamentadoras de pesquisas envolvendo seres humanos. Bioética. 1996;4(2 Supl):15-25.
As práticas de saúde determinam e são determinadas pelo cotidiano; portanto, não é possível afirmar que os trabalhadores sejam neutros e apenas executem o trabaIho, nas condições que Ihes são ofertadas/impostas; eles precisam ter consciência de que possuem um quantum de poder e governabilidade que mantém ou transforma a realidade.

12. Lima MADS, Almeida MCP. O trabalho de enfermagem na produção de cuidados no modelo clínico. Rev Gaúcha Enferm. 1999;20(n.esp):86-101.

13. Ludwig MLM, Bonilha ALL. O contexto de um serviço de emergência: com a palavra o usuário. Rev Bras Enferm. 2003;56(1):12-7.

14. Rosa NG. Dilemas éticos no mundo do cuidar de um serviço de emergência [dissertação]. Porto Alegre: Escola de Enfermagem, Universidade Federal do Rio Grande do Sul; 2001.

15. Oliveira MLF, Scochi MJ. Determinantes da utilização dos serviços de urgência/emergência em Maringá(PR). Ciênc Cuidado Saúde. 2002;1(1):129-34.

16. Giglio-Jacquemot A. Urgências e emergências em saúde: perspectivas de trabalhadores e usuários. Rio de Janeiro: FIOCRUZ; 2005.

17. Merhy EE, Cecílio LCO, Nogueira Filho RC. Por um modelo tecno-assistencial da política de saúde em defesa da vida: contribuição para as conferências de saúde. Saúde Debate. 1991(33):83-9.

18. Peduzzi M. Equipe multiprofissional de saúde: conceito e tipologia. Rev Saúde Pública. 2001; 35(1):103-9.

19. Gomes AMT, Oliveira DC. Estudo da estrutura da representação social da autonomia profissional em enfermagem. Rev Esc Enferm USP. 2005;39(2):145-53.

20. Gustavo AS, Lima MADS. Idealização e realidade no trabalho da enfermeira em unidades especializadas. Rev Bras Enferm. 2003;56(1):24-7.

21. Rodrigues FCP, Lima MADS. A multiplicidade de atividades realizadas pelo enfermeiro em unidades de internação. Rev Gaúcha Enferm. 2004;25(3):314-22. 